KINDER- UND JUGENDLITERATURFORSCHUNG 2001/2002 


\section{Kinder- und \\ Jugendliteraturforschung 2001/2002}

\section{Mit einer Gesamtbibliographie}

der Veröffentlichungen des Jahres 2001

In Zusammenarbeit mit der Gesellschaft für Kinder- und Jugendliteraturforschung in Deutschland und der deutschsprachigen Schweiz, der Österreichischen Gesellschaft für Kinder- und Jugendliteraturforschung und in Verbindung mit Verena Rutschmann (Zürich), Ernst Seibert (Wien)und Jack Zipes (Minneapolis)

herausgegeben von

Hans-Heino Ewers, Ulrich Nassen, Carola Pohlmann, Karin Richter und Rüdiger Steinlein

Verlag J. B. Metzler

Stuttgart - Weimar 
Redaktion: Bernd Dolle-Weinkauff, Hans-Heino Ewers (verantwortlich)

Rezensionen: Ulrich Nassen, Jan Bolczyk

Bibliographie: Karin Laubrecht

Layout: Hongqin Flicker, Antje Keil

Der Druck dieses Jahrbuchs wurde durch die Gesellschaft für

Kinder- und Jugendliteraturforschung in Deutschland und

der deutschsprachigen Schweiz gefördert.

Die Deutsche Bibliothek - CIP-Einheitsaufnahme

Kinder- und Jugendliteraturforschung ... :

mit einer Gesamtbibliographie der Veröffentlichungen des Jahres ... I

in Zusammenarbeit mit der Arbeitsgemeinschaft Kinder- und

Jugendliteraturforschung. - Stuttgart ; Weimar : Metzler.

Erscheint jährl. - Aufnahme nach 1994/95(1995)

ISBN 978-3-476-01926-4

ISBN 978-3-476-02891-4 (eBook)

DOI 10.1007/978-3-476-02891-4

Dieses Werk einschließlich aller seiner Teile ist urheberrechtlich geschützt. Jede Verwertung außerhalb der engen Grenzen des Urheberrechtsgesetzes ist ohne Zustimmung des Verlages unzulässig und strafbar. Das gilt insbesondere für Vervielfältigungen, Übersetzungen, Mikroverfilmungen und die Einspeicherung und Verarbeitung in elektronischen Systemen.

(C) 2002 Springer-Verlag GmbH Deutschland

Ursprünglich erschienen bei J. B. Metzlersche Verlagsbuchhandlung

und Carl Ernst Poeschel Verlag GmbH in Stuttgart 2002

www.metzlerverlag.de

info@metzlerverlag.de 


\section{Inhalt}

\section{Berichte}

Alice Ettwein: Internationales Kolloquium: Johanna Spyri und ihr Werk (Zürich 6.-7. Juli 2001)...3.-. Bernd Dolle-Weinkauff: CHILDE - Dokumentation des historischen Kinderbuchs im Internet ...7.-. Manuela Kocher: Workshop Raum und Zeit in interaktiven Kinder- und Jugendmedien (Zürich 23.-25.11.2001) ..99

\section{Beiträge}

\section{Ute Dettmar}

Docere - delectare - movere.

Zum Stellenwert der Unterhaltung in Poetik und Praxis kinderliterarischer Aufklärung

Susanne Pellatz

Predigt und Abenteuer. Zur Entwicklung der religiösen Unterhaltungszeitschrift

für Kinder und Jugendliche im 19. Jahrhundert

\section{Gisela Wilkending}

Massenunterhaltung und Jugendliteratur. Zur Auflösung

des narrativ-auktorialen Schreibkonzepts in der Jugendliteratur der Jahrhundertwende

Irmgard Wagner

Väter im Zwielicht. Der Ambivalenzeffekt bei Tony Schumacher.

\section{Ernst Seibert}

Das Experimentelle des Phantastischen: Der Surrealismus in der österreichischen

Kinderliteraturgeschichte.

Heinz-Jürgen Kliewer

Doppeladressierung in der Kinderlyrik.

\section{Forschungsberichte}

\section{Bernhard Rank}

Phantastik im Spannungsfeld zwischen literarischer Moderne und Unterhaltung.

Ein Überblck über die Forschungsgeschichte der 90er Jahre

Bernhard Rank / Martin-Christoph Just

Forschungsliteratur zu „Harry Potter“

Rezensionen 
Wie bereits in den vergangenen Jahren, versendet die Redaktion des Jahrbuchs an alle interessierten Bezieher kostenlos eine für IBM-kompatible PC-Systeme (MS-DOS/Windows) ausgelegte Datenbankversion der bisher veröffentlichten Jahresbibliographien (siehe Gutschein am Ende des Bandes). Da der Umfang der Bibliographie die Kapazitäten eine 1,44“-Diskette sprengt, werden seit der Ausgabe 1999/2000 CD-ROM an die Bezieher verteilt.

Anschriften der Herausgeber:

Prof. Dr. Hans-Heino Ewers, Institut für Jugendbuchforschung, der Goethe-Universität, Postfach 111932, 60054 Frankfurt/M.

Prof. Dr. Ulrich Nassen, Institut für Germanistik der Universität, Augustusplatz 9, 04109 Leipzig Carola Pohlmann, Kinder- und Jugendbuchabteilung der Staatsbibliothek zu Berlin, 10102 Berlin Prof. Dr. Karin Richter, Pädagogische Hochschule - Institut für Grundschulpädagogik und Kindheitsforschung, 99089 Erfurt

Prof. Dr. Rüdiger Steinlein, Humboldt-Universität, Philosophische Fakultät Il, Schützenstr. 21, 10117 Berlin 